\title{
Movimientos feministas en México: prácticas comunicativas digitales y riesgos
}

Sección: Dossier

Recibido: $17 / 08 / 2021$

Aceptado: 19/11/2021
Feminist Movements in Mexico:

Digital Communicative Practices and Risks

Lidia Ángeles García González

Universidad de Guanajuato, México

ORCID: 0000-0001-9363-5280

la.garciagonzalez@ugto.mx

In recent years, we have encountered the emergence of new interdisciplinary studies on feminist mobilizations in Latin America and the role played by Internet social networks. The main themes are the communicative practices of feminism, the shaping of new identities, technological appropriations, conceptual dimensions and perspectives, online feminist practices and/or strategic communicative use of digital tools in feminist mobilizations against gender violence, among others. The central objective is to respond from a sociocultural perspective on digital communication practices and the risks that are being generated in recent feminist mobilizations in Mexico. Methodologically this research, conducted through 16 interviews, proposes to communicate the dynamics around collective action in Mexico from the feminist collectives created between 2016 and 2021. It is a proposal focused on feminist agency in Internet's social networks for collective action that seeks to contribute to the void of academic literature on these recent feminist mobilizations in digital contexts in Mexico.

Key words: Cyber feminism, Feminist Digital Activism, Socio-digital networks.

Activismo Digital Feminista, Redes Sociodigitales.

\section{Abstract Introducción}

La persistencia de los feminicidios exhibe que las acciones siguen siendo escasas para prevenir, atender y sancionar la violencia contra las mujeres en México, 
la cual se encuentra situada en un contexto de violencia generalizada, discriminación e impunidad. El mes de abril de 2020 ha sido considerado el más violento para las mujeres en México: en términos de feminicidios se registraron 339 muertes violentas; esto se traduce en más de 11 feminicidios al día. Asimismo, hubo un incremento de $19.2 \%(13,317)$ de presuntos delitos de violencia familiar y de $18.0 \%(208)$ en todas sus modalidades entre enero-abril de 2020 y enero-abril de 2021 , y, en el mismo periodo, los presuntos delitos de violación aumentaron $21.4 \%(1,175)$.

En el periodo entre el 1 de enero de 2018 y el 20 de junio de 2021 el registro nacional de personas desaparecidas y no localizadas (RNPDNO) documentó la desaparición de 25,340 mujeres, niñas y adolescentes en México. De la misma manera, la SESNP indica que entre enero y diciembre de 2020, 10.2 mujeres y niñas fueron asesinadas por día en México. El 2020 cerró con el mayor número de denuncias por violencia familiar desde que se tiene registro: 220,028, según el reporte actualizado del Secretariado Ejecutivo del Sistema Nacional de Seguridad Pública (SESNSP).

Es decir, que se abrieron en promedio 603 carpetas de investigación al día, 25 cada hora del año. Finalmente, el panorama mexicano para las mujeres en México es desalentador ya que el gobierno federal realizó un recorte de más 37 millones de pesos al presupuesto otorgado a la Alerta de Violencia de Género contra las Mujeres en las entidades que más feminicidios registraron en el 2020: Estado de México, Veracruz, Nuevo León, Ciudad de México, Puebla, Jalisco, Nayarit y Zacatecas. Y en ese mismo año, el gobierno recortó 1,872 millones de pesos de 20 programas de apoyo a las mujeres (Mi punto de vista, 2020). Ante este escenario de creciente violencia en los últimos años contra las mujeres en México ha habido un incremento en la creación de colectivas feministas en diversas partes del país para atender la problemática y emergencia humanitaria que cotidianamente aqueja a miles de mujeres en México.

En este sentido, la pregunta que guía esta investigación es la siguiente: ¿cuáles son las prácticas comunicativas digitales que utilizan para aprovechar las fortalezas del activismo digital y sus riesgos en México ante esta problemática social? Para ello, presentamos a continuación una revisión de literatura que destaca los aspectos más importantes del ciberactivismo feminista, así como las implicaciones del cambio tecnológico para luchar por las erradicación de la violencia contra las mujeres que ha permitido la multiplicación de diversas prácticas comunicativas feministas a través de las plataformas digitales, lo que potencialmente abre la posibilidad de convertir al feminismo en un movimiento inclusivo sin que exista una militancia dentro del mismo, sino a partir de tener un sentido de interpelación sobre las posibilidades que brindan las interconexiones digitales para la visibilización de esta problemática. Sin embargo, al mismo tiempo, hay una tensión ante la existencia de la llamada brecha digital que excluye a miles de mujeres que no tienen acceso a Internet y a la alfabetización digital para el manejo de las herramientas, lo que denota el privilegio de las mujeres que pueden participar y organizarse; es decir, un reto a futuro para la democratización de los medios de movimientos feministas en México.

\section{El feminismo de la cuarta ola y ciberactivismo feminista}


El feminismo de la cuarta ola se caracteriza por el cambio tecnológico y las posibilidades que Internet permite para hacer un híbrido de las prácticas feministas con más fuerza y convertirlo en popular y reactivo. Varela (2020) sostiene que el movimiento feminista produce una novedosa acción: "la de las multitudes anónimas organizadas de forma rápida y precisa, con objetivos claros y comunes, con una estrategia que puede discutirse y planificarse" (p. 106), y, una vez que las acciones se llevan cabo en el espacio digital, potencia la permanencia de las interconexiones virtuales que brindan conciencia a colectivas cada vez más jóvenes e interconectadas a una escala global. Asimismo, una de las características más importantes de la cuarta ola del feminismo según Varela (2020) ha sido "la diversidad de las mujeres y se ha asentado esta idea en su configuración ideológica, de manera que ya es posible desplazar el foco desde el interior del feminismo hasta fuera, hasta los fenómenos sociales patriarcales más opresivos" (Varela, 2020, p. 102). Asimismo, una de las preocupaciones que el ciberactivismo feminista ha cultivado en relación a la representación simbólica es referente a la violencia de género en diversos discursos mediáticos (Núñez, Vázquez y Fernández, 2016).

Twitter, por ejemplo, ha sido la plataforma social más relevante para el activismo feminista de la cuarta ola, debido a la expansión de la interseccionalidad. "Identificar el privilegio, la diferencia, la representación y el racismo desde un enfoque interseccional es un prerrequisito necesario para las feministas de la cuarta ola" (Zimmerman, 2017, p. 64). Desde la virtualidad se pueden conformar colectivas, acercarse a colectivas en las universidades, o bien, adherirse al movimiento feminista organizado; en síntesis, la virtualidad ha dado paso a "un nuevo espacio de opinión pública al que las mujeres nunca habían tenido acceso por el control patriarcal de los medios de comunicación" (Varela, 2020, p. 106).

Recientemente, la apropiación de redes sociodigitales por parte del movimiento feminista en México se ha focalizado en combatir $u$ oponerse al establecimiento de un feminismo institucional que se arraiga a los mandatos del sistema capitalista neoliberal. En consecuencia, "el activismo feminista digital resurge como un contrapoder que se enfrenta a la lógica mercantilista del neoliberalismo y las desigualdades de género que reproduce dicho sistema" (Reverter y Medina, 2020, p. 75). Desde la perspectiva de Revilla (2019), el movimiento feminista en la región ha pasado por dos procesos importantes en la última década:

1) [...] la diversificación de las identidades feministas con la mayor visibilización de las identidades étnicas y de las identidades de género (desde las mujeres indígenas y afrodescendientes a los feminismos lésbicos y transgénero) y 2) el aumento de la participación digital individual y colectiva y sus efectos posibles en la ampliación del repertorio de movilización y de su difusión con nuevas herramientas (app, producción cultural autónoma, etc.) ha facilitado la movilización en una pluralidad de espacios, desde las manifestaciones, concentraciones y caceroladas en la calle hasta las producciones audiovisuales, pasando por la difusión y el alcance de los mensajes en campañas virtuales (Revilla, 2019, p. 49). 
Asimismo, el movimiento ciberfeminista latinoamericano se caracteriza por la adopción de una organización horizontal; apropiación de prácticas de contención políticas, por ejemplo, acciones directas creativas o la creación de imaginarios radicales; uso intensivo de redes sociodigitales como medio, objeto de crítica y transformación. Binder (2019) explica que:

Un movimiento que extiende lazos difusos con el movimiento hacker y de software libre, por un lado, y con el movimiento feminista y de justicia global, por el otro. $Y$ que, sin dirigir necesariamente su política contenciosa a instituciones del Estado o a través de manifestaciones públicas masivas, desafían el status quo con formas de resistencia discursivas y culturales (Binder, 2019, p. 217).

El estudio del ciberactivismo feminista da cuenta de las potencialidades para la acción colectiva mediante las plataformas digitales para la disputa por los derechos, visibilización de temáticas y organización de la acción colectiva en el espacio público tradicional. Acosta y Lassi (2020) aseveran que ante el conocimiento de las diversas "estrategias y sus tácticas se pueden [...] gestar y consensuar en las plataformas digitales pero las revoluciones se hacen en las calles poniendo el cuerpo. De allí la importancia que tiene la construcción y circulación de los marcos interpretativos" (Acosta y Lassi, 2020, p. 104). Ha sido a través del paso del tiempo mediante protestas y denuncias que las ciberactivistas han logrado "desarrollar un proceso de concienciación de la sociedad civil que ha permitido resituar la violencia machista como problema social y no individual” (Sundén y Paasonen, 2019).

Finalmente, el hecho de que el feminismo utilice medios de comunicación de masas lo transforma en popular, lo hace expansivo; un público mayor o diversos grupos pueden sentirse identificados con el feminismo; "estos feminismos, al ser populares, se desarrollan en un contexto de lucha, de resignificación de relaciones de poder donde unos significados luchan por imponerse a los otros" (Reverter y Medina, 2020, pp. 69-79).

\section{Apuntes sobre las principales características del movimiento feminista en México}

El pasado 9 de marzo de 2019 se realizó un paro a nivel nacional denominado \#UnDíaSinNosotras donde se estima que participaron 22 millones mujeres mexicanas (Ayala, 2021), que dio cuenta de la existencia del potencial organizativo de las mujeres en México.

En tal sentido, Álvarez-Enríquez (2020) describe que algunas de las características que fortalecieron el movimiento feminista en el 2019, fueron (1) el surgimiento de nuevas actoras, (2) el lenguaje novedoso, (3) las estrategias de acción, (4) la alfabetización en el uso de herramientas digitales y (5) las demandas auténticas, dado que son parte de una nueva generación. Cerva-Cerna (2021) 
expresa que la protesta feminista es la reacción ante la impunidad y revictimización. Para Castellanos (2020), las nuevas actoras

No son feministas teóricas sino vivenciales. Se apropiaron de un término históricamente estigmatizado y lo resignificaron y han hecho popular movidas por la rabia ante las violencias machistas, y por la sororidad (hermandad solidaria femenina) que a partir de la violencia han ido construyendo con las mujeres de su familia, su entorno y su país (Castellanos, 2020, en línea).

Actualmente, las acciones que las mujeres llevan a cabo en la calle representan una oportunidad para la lucha por la igualdad de género, a que "muchas mujeres jóvenes sin militancia en el feminismo se hayan sentido interpeladas y atraídas a decir y a hacer, a ser protagonistas de una nueva ola global de movilizaciones que adquieren la forma de constelaciones performativas claramente feministas" (Rovira-Sancho, 2018, p. 228).

En el caso mexicano, las acciones que han llevado a cabo las jóvenes mexicanas han consistido en protestas en las calles, performances artísticos, cristalazos y pintas de monumentos icónicos, toma de planteles educativos para exhortar el despido de acosadores sexuales, la destrucción o incendio de oficinas públicas y la obstrucción de calles, por lo que han sido llamadas "vándalas" y acusadas de haber sido infiltradas por la derecha por el presidente del país (Castellanos, 2020). El movimiento feminista en tal sentido está logrando mucha visibilidad y cobertura mediática (Pfleger, 2021). Para Álvarez-Enríquez (2020) las principales causas que han derivado en el movimiento feminista hoy día son tres:

a) el aumento generalizado de la violencia en nuestro país, y en particular la violencia contra las mujeres, b) la impunidad en el tratamiento de los delitos de género, la ineficacia de la justicia y la "normalización" de esta situación, y c) la expansión de una animadversión cada vez mayor de amplios grupos de hombres contra las mujeres, de un fuerte resentimiento e incluso de un odio manifiesto ante la creciente autonomización y empoderamiento de éstas, que se ha traducido en una suerte de "ánimo vengativo" (Álvarez-Enríquez, 2020, p. 150).

Otra de las características más notorias del movimiento feminista es su diversificación de las identidades, ya que "es uno de los desarrollos más importantes en los últimos años porque representa una toma de postura de auto reconocerse y auto constituirse" (Pfleger, 2021, p. 330). En lo referente a la diversidad de las colectivas feministas a partir del principio de sororidad que se deja manifiesta de las mujeres más jóvenes con las mujeres mayores, esto se expresa en que las protestas se llevan de manera solidaria entre: "hijas, madres, estudiantes, artistas y trabajadoras. Hay una aumentada presencia de mujeres indígenas que marchan junto a mujeres de clase media y de mujeres que vienen desde lugares periféricos de la ciudad y de los Estados de la República" (p. 337). 
La autora sostiene que este aumento en la violencia de género -en específico de los feminicidios - se debe precisamente a que en "el aparato jurídico e institucional existente los agresores saben bien que 'no pasa nada', y en última instancia se harán acreedores a sólo un castigo menor" (Álvarez-Enríquez, 2020, p. 154), y que la impunidad es una de las causas que más afecta a las mujeres que han padecido dicha violencia: "al mismo tiempo es uno de los desencadenantes de la rabia y de la movilización en curso" (p. 154).

Días después de la marcha \#8M y el \#9M se dio el confinamiento en México debido a la pandemia del Covid-19, que obstaculizó la continuidad del movimiento en el espacio público, sin embargo, con otras dinámicas en las redes sociales de Internet, "esto deja ver que la potencia del movimiento se mantiene activa" (ÁlvarezEnríquez, 2020, p. 170). El movimiento feminista en México, durante el actual gobierno del presidente Andrés Manuel López Obrador, ha fungido como oposición "por su capacidad de cuestionar la actuación e indiferencia de la actual administración que prometía cambios estructurales y mayor respeto a los derechos humanos de toda la población" (Cerva-Cerna, 2021, p. 123).

Cerva-Cerna argumenta que el movimiento feminista en México enfoca su demanda en cuestionar la ausencia de acciones y justicia "por parte de las autoridades y las constantes situaciones de revictimización y violencia institucional, también presente en los medios de comunicación y su extensión en las redes sociales" (Cerva-Cerna, 2020, p. 179). A esta problemática la autora la denomina: 'doble indignación', y la ubica en el centro como el principal insumo del movimiento feminista en el país. Cerva-Cerna (2020) sustenta que las feministas mexicanas ante las redes sociales de Internet han perdido el miedo para externar sus sentimientos de identificación con una "lucha emancipatoria de carácter global" (p. 179).

Asimismo, el lenguaje que emplean las jóvenes feministas mexicanas se caracteriza por ser más directo y controversial, lo que genera un efecto notable en "la opinión pública, y provoca de manera más directa la atención de las autoridades exigiendo al Estado-nación con mayor fuerza el aseguramiento de los derechos de igualdad para las mujeres en el país" (Pfleger, 2021, p. 329).

\section{Aproximaciones sobre el papel de las redes sociodigitales en la práctica del activismo digital feminista}

Uno de los más representativos ejemplos de la lucha feminista actual —bajo un contexto de redes sociodigitales - se ha dado en los últimos cinco años, durante los cuales América Latina ha jugado un papel clave en cuanto a movilizaciones masivas, como con el movimiento \#NiUnaMenos, en Argentina, a raíz de los feminicidios y la violencia contra las mujeres, que ha tenido eco en diversos países del continente, diversificando colectivos y organizaciones colocando al centro las voces de las mujeres (Larrondo y Ponce, 2019).

Las redes sociodigitales han jugado un papel fundamental en el quehacer del activismo digital feminista a partir de que le ha provisto de mayor participación en la agenda pública y un lenguaje propio (Piñero-Otero, 2021), brindando "a las participantes en dicho movimiento de las herramientas precisas para el lanzamiento, 
difusión y adhesión de sus demandas o protestas sociales y políticas" (Piñeiro y Martínez, 2016, p. 19). Por otro lado, según Reis y Natansohn (2019, p. 394) la "hiperconexión mundial" a partir de las plataformas digitales, ha permitido "la multiplicación de grupos y redes feministas" y a través del uso de hashtags se han podido organizar con objetivos comunes y captar un notable control del propio discurso, esto es, de la voz e imagen de los distintos movimientos de mujeres, y tener más agencia para la conformación de la propia identidad (Pfleger, 2021).

Dentro de las prioridades de las activistas feministas "las prácticas en la red se convierten en una característica clave de estas organizaciones, su sentido de pertenencia al feminismo como movimiento social y quién está excluido de la identidad feminista, que a menudo se realiza en línea" (Fotopoulou, 2016, p. 993). En otros términos, Pedraza y Rodríguez (2019) acuñan el término 'tecnopolítica feminista' para dar cuenta de la revalorización de la relación de las mujeres con la tecnología en la diversidad de los espacios, así como con "las tecnologías de la reproducción, el cuidado, la alimentación, la creación artística; es decir, recuperando la dimensión política de la vida cotidiana" (Pedraza y Cano, 2019, p. 203).

Por otra parte, según Alvarado et al (2020, p. 12), el discurso feminista entrelaza lo "íntimo-privado-político", habitando el espacio público como territorio común de (des)encuentros, de reclamos, denuncias y malestares.

Asimismo, las herramientas digitales permiten la circulación de información sobre sus acciones "a través de convocatorias para narrar experiencias de violencia, escrachar a acosadores, publicitar talleres de autodefensa, conversatorios sobre la temática, festivales culturales e intervenciones artísticas o pintas" (Di Napoli, 2021, p. 13).

En el espacio digital, se puede compartir blogs, podcasts o listas de difusión a través de la creación de diversos tipos de contenidos, que tienen relación "a la teoría y praxis feministas, a la presentación de voces y perspectivas más diversas y disidentes" (Piñero-Otero, 2021, p. 234), misma información que la mayoría de las veces es diseñada y producida por ellas mismas, o bien se apoyan en una diseñadora o diseñador; estos sitios de redes sociales se ha vuelto relevantes para que estas mujeres "puedan difundir su trabajo; ya sea en redes sociodigitales, plataformas que alojan audios, videos o imágenes, o en sitios web de ellas mismas y de otras colegas, estas mujeres difunden las actividades y productos que realizan" (Becerril, 2019, p. 23). A raíz de la pandemia del Covid-19, el fenómeno comunicativo de los podcasts feministas se ha expandido convirtiéndose en un

Nuevo medio en un canal para "tomar la voz" y desarrollar su práctica activista a través de iniciativas de automediación. Basculando entre el educar, informar y entretener, dichas iniciativas desarrollan una importante labor en la proyección social de temáticas, enfoques y protagonistas obviados por los medios de comunicación mainstream, pero también detentan una función identitaria que posibilita la construcción del "nosotras" (Piñero-Otero, 2021, p. 246). 
Este tipo de productos abiertos hacen un frente común que genera prácticas colaborativas para posicionar determinadas perspectivas de interés feminista, como la red mundo nosotras en él (Piñero-Otero, 2021, p. 253).

\section{Algunas consideraciones sobre los riesgos de las prácticas comunicativas del activismo digital feminista}

A lo largo de la historia el feminismo como movimiento social -explica Mazón (2021) - se ha encontrado con variados inconvenientes, principalmente, expresiones patriarcales en las redes socio digitales. Cerva-Cerna (2020) argumenta que coexisten dentro de la lucha feminista "escenarios de acoso y violencia; ante el ciberactivismo feminista ha surgido una serie de hostigadores que tienen como fin desvirtuar el sentido de las demandas feministas" (p. 200). Banet-Weiser y Milter (2016) han presenciado un incremento de una fuerte misoginia como resultado de toda una configuración establecida, símbolos y lógicas de orden patriarcal que hoy en día siguen vigentes circulando por las redes sociodigitales. En lo referente a las estrategias para combatir la violencia digital las activistas incluyen:

Ignorar el comentario, bloquear, cancelar la amistad, responder (como individuos o comunidad), denunciar su experiencia a la policía y generar nuevas iniciativas para apoyar a las víctimas del sexismo en línea. Aunque "borrar", "bloquear" o "eliminar de la lista de amigos" se ha sido visto negativamente como una forma de censura por anteriores activistas feministas. ${ }^{1}$

Ante este panorama las colectivas feministas, requieren de idear prácticas y estrategias de resistencia a las reconfiguraciones que asume la violencia digital. Reis y Nathansohn (2019) exponen la presencia de "fenómenos como ciberchantaje, divulgación de datos e imágenes sin consentimiento, trolls, y todo tipo de ataques misóginos, son algunos de los desafíos que crecen proporcionalmente a las oportunidades generadas por las tecnologías" (Reis y Natansohn, 2019, p. 394). En la pasada convocatoria del \#8M de 2020 en México, según Villar-Aguilés y Pecourt (2021), se dio un ataque organizado al movimiento feminista, "incluso, la culpabilización de la expansión de la pandemia del coronavirus. Estos hechos sugieren una interesante línea de investigación futura para seguir profundizando en el troleo antifeminista como fenómeno social" (p. 43). Pedraza y Rodríguez (2019) proponen la necesidad de la autodefensa digital al centro proveniente de la tecnopolítica feminista con la finalidad de suscitar

Aprendizajes que reconozcan la capacidad de acción de las mujeres para resolver los problemas apremiantes de su entorno. Particularmente, dado el

\footnotetext{
${ }^{1}$ ["ignoring the comment, blocking, unfriending, responding (as individuals or a community), reporting their experience to the police, and generating new initiatives to support victims of online sexism. Although "deleting," "blocking," or "unfriending" has been viewed negatively as a form of censorship by previous feminist activists"] (Mendes et al., 2019, p. 92).
} 
contexto de violencia de género (digital y fuera de línea), dotar de herramientas y tácticas para enfrentarla a través de las tecnologías resulta fundamental para garantizar su participación pública (p. 209).

En "Efectos de la pandemia por la Covid-19 en las movilizaciones feministas de la Ciudad de México", de Portillo y Beltrán (2021), se concluye que las movilizaciones feministas se caracterizan por construir 'redes de protección y cuidado'. Las autoras identifican una serie de estrategias para mantener el resguardo de "las integrantes de colectivas tanto en los espacios físicos como digitales" (Portillo y Beltrán, 2021, p. 30), y, por otro lado, en el contexto de la pandemia del Covid-19 la seguridad digital con perspectiva de género se ha convertido y posicionado como un tema prioritario y búsqueda de soluciones.

Además, el activismo digital feminista constituye en sí mismo una actividad con un alto 'agotamiento emocional' y bajo el contexto del confinamiento se ha incrementado, de tal manera que Portillo y Beltrán (2021) explican que una de las prácticas que han implementado al interior de las colectivas ha sido "la vigilancia con ellas mismas y con sus compañeras" (p. 30). Para Mendes et al., (2019, p. 74) el activismo digital feminista puede ser "altamente explotador" y, en muchas ocasiones, sin compensación económica. En tanto a las actividades que realizan en los sitios web, se dio cuenta de que el trabajo requería mucho tiempo y con frecuencia suele ser muy tedioso y, como se ha indicado anteriormente, "emocionalmente agotador". En consecuencia, las activistas han optado por prácticas para lidiar con el agotamiento, como "tocar música, la meditación, el yoga, la lectura de obras feministas, los paseos por el parque, la participación en comunidades feministas, tomarse un descanso de su activismo o de sus actividades en línea" (Mendes et al., 2019, p. 97).

Otro de los riesgos del activismo digital feminista, según Mazón (2021), es que no se contemplan la diversidad de formas de vida de las mujeres. Este autor sostiene que el movimiento feminista en las redes sociodigitales implica "una cuestión de privilegio", ya que se excluye a las mujeres que no tienen acceso a Internet y a las que no tienen los conocimientos y destrezas para el manejo y uso de las herramientas digitales y "a todas las mujeres que no recibieron educación porque no les era permitido y no saben leer o escribir" (Mazón, 2021, p. 38). En el caso de las jóvenes activistas estudiantiles no ha construido una estrategia prioritaria para expandir al movimiento a otros sectores sociales (Cerva-Cerna, 2021). Finalmente, Laudano (2019) indica que dentro de las limitaciones del activismo digital feminista se encuentran "las competencias comunicativas, así como a la democratización del acceso a la información y el uso de la palabra, ya que se pueden destacar las voces de ciertos grupos o personas con mayores habilidades y capital social" (pp. 69-70), colocando en riesgo de minimizar determinadas acciones colectivas y las trayectorias vitales de otros sectores del movimiento de mujeres y feminista.

\section{Metodología}


El propósito de este artículo tiene como objetivo examinar las prácticas comunicativas digitales y sus riesgos para la acción colectiva por parte de coaliciones feministas que

\section{Tabla 1.} Información de las activistas entrevistadas

dan sentido al movimiento, mediante los discursos que articulan contra la violencia de género y conocer cuáles son los repertorios de acción colectiva digital empleados. En tal sentido optamos por el uso de las entrevistas cualitativas que pueden ser una ventana al mundo cotidiano de los activistas y generan representaciones que encarnan las voces de los sujetos, minimizando - al menos en la medida de lo posible - la voz del investigador (Ragin y Amoroso, 1994). Para Klandermans y Staggenborg (2002, pp. 95-96), la utilización de entrevistas para investigar los movimientos sociales ofrece la oportunidad de conocer "el contexto de las motivaciones, creencias y actitudes de los participantes en los movimientos sociales". Además, permite investigar al "individuo y sus visiones colectivas, imaginaciones, esperanzas, expectativas, críticas del presente y proyecciones del futuro sobre las que descansa la posibilidad de acción colectiva y a través de las cuales los movimientos sociales se forman, resisten o se dispersan".

James y Busher (2012) indican que las plataformas para video llamadas pueden ser usadas con propósitos de investigación, y también subrayan cómo los sitios de redes sociales, como Facebook, están siendo explorados ahora como un lugar donde se pueden realizar entrevistas "en vivo", en lugar de enviar preguntas por correo electrónico, considerando que, actualmente -ante el contexto de la pandemia global por el Covid-19_, plataformas como Zoom, Google Meet, Jitsi Meet y Teams proporcionan nuevas posibilidades para llevar a cabo entrevistas en línea, y cuentan con la ventaja adicional de que permiten grabar las sesiones.

Con el propósito de este estudio se realizaron 16 entrevistas con activistas feministas de nueve colectivas mexicanas entre el mes de noviembre 2020 y febrero 2021. El muestreo se construyó, vía correo electrónico, de un universo de 35 colectivas feministas en México considerando que la creación de la colectiva hubiera sido dentro del periodo que del 2016 al 2020 y que tuvieran página en Facebook 


\begin{tabular}{|c|c|c|c|c|}
\hline $\begin{array}{l}\text { Nombre de la } \\
\text { colectiva y } \\
\text { activista }\end{array}$ & $\begin{array}{l}\text { Año de } \\
\text { formación }\end{array}$ & Profesión & $\begin{array}{l}\text { Lugar de } \\
\text { residencia }\end{array}$ & Edad \\
\hline $\begin{array}{c}\text { 1.Las del } \\
\text { Aquelarre } \\
\text { feminista } \\
\text { (Ana Elena } \\
\text { Contreras) }\end{array}$ & 2016 & Abogada & CDMX & 44 \\
\hline $\begin{array}{l}\text { 2. Brujas del Mar } \\
\text { (Arusi Unda) }\end{array}$ & 2019 & Mercadóloga & Veracruz, Ver. & 32 \\
\hline $\begin{array}{l}\text { 3. Colectiva } \\
\text { Artemisas } \\
\text { Veterinarias } \\
\text { (Anónimas) }\end{array}$ & 2020 & $\begin{array}{l}\text { Estudiantas } \\
\text { médica } \\
\text { veterinaria }\end{array}$ & CDMX & $\begin{array}{c}34,21,19,21 \\
\text { y } 21\end{array}$ \\
\hline $\begin{array}{l}\text { 4. Igualdad } \\
\text { Sustantiva } \\
\text { Yucatán } \\
\text { (Kelly Ramírez) }\end{array}$ & 2018 & Psicóloga & $\begin{array}{l}\text { Mérida, } \\
\text { Yucatán }\end{array}$ & 30 \\
\hline $\begin{array}{l}\text { 5. Red de } \\
\text { Movimientos } \\
\text { Feministas } \\
\text { (Patricia } \\
\text { Rodríguez) }\end{array}$ & 2019 & Pedagoga & $\begin{array}{l}\text { León, } \\
\text { Guanajuato }\end{array}$ & 45 \\
\hline $\begin{array}{l}\text { 6. Siempre Unidas } \\
\text { (Tania Ramírez) }\end{array}$ & 2017 & $\begin{array}{l}\text { Lic. en } \\
\text { Lenguas }\end{array}$ & $\begin{array}{c}\text { Cancún, } \\
\text { Quintana Roo. }\end{array}$ & 41 \\
\hline $\begin{array}{l}\text { 7. Luchadoras } \\
\text { (Lulú Barrera) }\end{array}$ & 2012 & $\begin{array}{l}\text { Lic. en } \\
\text { Letras } \\
\text { Españolas }\end{array}$ & CDMX & 39 \\
\hline $\begin{array}{l}\text { 8. Abogadas con } \\
\text { Glitter } \\
\text { (Anónimas) }\end{array}$ & 2019 & $\begin{array}{l}\text { Estudiantas } \\
\text { en Derecho }\end{array}$ & CDMX & $\begin{array}{c}26,23,28 y \\
28\end{array}$ \\
\hline $\begin{array}{c}\text { 9. Restauradoras } \\
\text { con Glitter (Sofía } \\
\text { Riojas) }\end{array}$ & 2019 & Arquitecta & CDMX & 33 \\
\hline
\end{tabular}

(de manera previa, su correo se obtuvo de su página de Facebook). Cada entrevista tuvo una duración de entre 40 minutos y 1:30 hrs., y fue videograbada en línea mediante la plataforma Google Meets. Asimismo, se les pidió mantener comunicación vía WhatsApp o correo electrónico después de la entrevista, por si fuera necesario dar seguimiento a la entrevista. Asimismo, se les preguntó a las participantes si preferían proporcionar su nombre o mantenerse en el anonimato; para el presente estudio, hubo dos colectivas feministas que eligieron no proporcionar su nombre por mantener su seguridad. 


\section{Resultados. Principales prácticas comunicativas en las redes sociodigitales de las colectivas feministas en México.}

En este estudio ubicamos el punto de partida del activismo digital feminista en la colectiva "Las del Aquelarre Feminista" y "Luchadoras". La trascendencia de la denominada primavera violeta —una movilización nacional contra las violencias machistas que se realizó el 24 de abril de 2016, a través de la campaña \#NoTeCalles y \#VivasNosQueremos, donde se emplearon diferentes hashtags que lograron las principales tendencias en las redes sociales de Internet- logró visibilizar el problema de la violencia y el riesgo de la vida de las mujeres y las niñas, principalmente en el espacio público.

Nosotras comenzamos a hacer por ejemplo lo que te mencionaba del \#24A ese primer ejercicio que hicimos fue completamente en redes sociales y tenemos unas reuniones así kilométricas en ese entonces no había tanto esto de este tipo de herramientas como el Google Meet o Zoom sino que lo hacíamos todo por, por este, por grupos de chats y así fue como nos estuvimos organizando para todo lo demás (Ana Elena, Colectiva Las del Aquelarre Feminista).

Para la colectiva Brujas del Mar, de Veracruz, el teléfono móvil es la herramienta esencial que les permite mayor acción en su quehacer activista: "[...] porque a través de él nos avisan de todo y con lo que podemos ayudar y con lo que podemos hacer contacto con otras colectivas, con víctimas [...] sin duda, puedes hacer todo desde ahí" (Arusi Unda, Brujas del Mar). En el contexto de la pandemia del Covid-19, el activismo feminista identificó la necesidad de adoptar las herramientas digitales en su quehacer cotidiano dado el confinamiento, lo que les permitió:

[...] hacer como enlaces con otras organizaciones aunque no sean de la Ciudad de México, no es que tengamos como una colectiva hermana con la gente estamos participando pero si colaboramos en ciertas ocasiones o coincidimos con varias organizaciones tan es así que una colectiva de Ecuador nos ha apoyado a hacer una campaña de Twitter o mujeres de Tabasco nos han contactado ahora la era digital, nos ha alcanzado [...] esto de la cuarentena es una manera de llegar como a más sitios en menor tiempo e ir conociendo las luchas de otras mujeres, y acuerparnos entre todas (entrevistada anónima, Colectiva Artemisa Veterinarias).

La pandemia hizo que las personas estén más enfocadas también en lo digital y también, por ejemplo, durante esta pandemia ha crecido demasiado el número de seguidores de la página, y [en] el número de seguidores de los eventos en vivo sí ha habido un aumento qué tiene que ver [...] precisamente con el aislamiento y la pandemia, porque hemos aprendido a manejar las 
herramientas que tienen que ver con la digitalidad (Kelly Ramírez Alpuche, Igualdad Sustantiva Yucatán).

Las prácticas comunicativas en Internet y las redes sociodigitales más recurrentes que realizan las colectivas feministas entrevistadas son: "ilustraciones con contenido, con mensajes, infografías, realizamos comunicados, dependiendo a veces realizamos comunicados, los en vivo también, o sea, los live, como videoconferencias; invitamos a otras feministas, a veces publicamos opiniones también en todas las plataformas" (Kelly, Igualdad Sustantiva Yucatán).

Y también dan cuenta de la necesidad de profesionalización en el manejo de las redes sociodigitales para que el acompañamiento y las canalizaciones se realicen óptimamente. Otra de las razones que consideran las colectivas crucial es la apropiación de las redes sociodigitales, debido al potencial que brindan estas herramientas para visibilizar

[...] todos los problemas que se están viviendo de manera inmediata a partir de las transmisiones "en vivo" [...] y llegar a muchas más personas; he tenido entrevistas de gente de Italia, de Francia, y otras partes, que a veces están mucho más interesadas y mucho más preocupadas por lo que está pasando aquí que la misma comunidad (Tania, Siempre Unidas).

Para la colectiva Luchadoras, Internet les ha posibilitado realizar activismo mediático, en específico, storytelling digital feminista que consiste en "contar historias transformadoras porque creemos en el poder transformador de las mismas", y, en segundo lugar, se enfocan en el ciberfeminismo que comprender y politizar el uso de Internet estratégicamente a partir de

Apropiarnos de las tecnologías, a las mujeres nos han dicho que somos usuarias de Internet, pero también somos creadoras y, entonces, también hemos dado talleres muy bonitos de creación a través de las tecnologías para que otras mujeres también se animen a tomar la cámara a, contar sus propias historias. Hemos hecho unos talleres muy divertidos, por ejemplo, de mi feminista qué nos parece que el meme y el gif feminista como una forma de comunicación muy contraria muy pícaras que a través de la risa y sin tanta seriedad puede comentar temas políticos muy actuales, y la risa es un gran mecanismo para contrarrestar y combatir al poder (Lulú Barrera, Colectiva Luchadoras).

En el caso de la colectiva Abogadas con Glitter, Internet y las plataformas como Facebook, WhatsApp y el correo electrónico están en la base para poder llevar a cabo su labor de brindar asesorías y compartir información en las redes sociodigitales lo expresan manifestando que:

Internet es una de las vías existencia de la colectiva [...] con esta realidad de la pandemia creo que lo ha dejado muy claro en muchos aspectos y en estas 
redes de apoyo feministas si no fuera por estas aplicaciones sería muy complicado porque no podemos tener actividades presenciales, pero muchísimo antes de este contexto de la pandemia, desde una página de Facebook nos da mucho porque son nuestras herramientas de día a día para realizar nuestras labores (Abogadas con Glitter).

Finalmente, para la colectiva Restauradoras con Glitter, es desde el mismo espacio digital que se conformaron y publicaron sus postulados, y manifiestan que es en Internet donde han hecho sus contactos "con las otras colectivas en diferentes redes sociales, y desde ahí hemos contactado, pues a la mayoría de los medios tanto nacionales como internacionales - con los cuales tenemos, al día de hoy, todavía, como..., mucha relación" (Restauradoras con Glitter).

\section{Principales hallazgos sobre los riesgos y limitaciones en el activismo digital feminista en México}

Como expusimos anteriormente existen diversos riesgos y limitaciones en las prácticas feministas en el espacio digital debido a una fuerte carga misógina y machismo en las que las colectivas feministas en México se han visto amenazadas en su integridad física y emocional como es el caso de Ana Elena, de la colectiva Las del Aquelarre Feminista, quien comenta:

Yo estoy ahorita en un proceso legal porque después del $1^{\circ}$ de noviembre de 2020, que tuvimos la marcha de las catrinas, debido a una publicación que hice el día 2 de noviembre en la mañana doxearon mi perfil y sacaron un montón de información y empecé a recibir amenazas de muerte, violación: que me iban a golpear si me encontraban en la calle, a exhibir dónde vivo y también estuvieron hostigando a mi jefe para que me despidiera del Congreso y tuve que levantar una denuncia en la fiscalía especializada para Defensores de Derechos Humanos y acogerme a tal mecanismo para poder tener un acompañamiento y yo siempre estuve pensando en todo este tiempo que yo era de muy bajo perfil (Ana Elena, Las del Aquelarre Feminista).

Una de las preocupaciones de las colectivas es el manejo y comunicación de información en sus plataformas digitales donde expresan que tienen que emplear protocolos y una básica para evitar conflictos que puedan colocar su integridad física en riesgo manifestando la existencia de:

Reglas simples de hasta donde puedes decir las cosas, [...] hasta donde puedes llegar sin meterte en tantos problemas; esas a nosotras nos las enseñaron cuando fue lo del paro nacional \#9M en el 2020, justamente para no meternos en problemas serios. Puedes ser muy crítica en cuestionar todo; tienes que saber cómo, Señalar así nada más por así, dar nombres, así como así; por supuesto que no puedes mencionar nada que tenga que ver con el crimen organizado [...] nosotras ahí sí es consigna, ahí no nos metemos, 
vivimos en Veracruz, o sea, ¡imagínate, ni de broma! ¡Vaya! Una cosa es ser valiente y otra tonta ¿no? (Arussi Unda, Brujas del mar).

Varias colectivas feministas mencionan que es importante mantener el anonimato para mantenerse a salvo ante la cacería de mujeres, sin embargo, el riesgo es que no se reconozca el trabajo realizado. Asimismo, otro riesgo manifestado es la censura ante la publicación de determinados contenidos, esto generando que les puedan retirar el uso de página en redes sociales como Facebook; y también existe la censura ante el uso de determinadas fotos expresan.

Nos pasó que, en una denuncia, nos sancionaron porque era una foto en la que un tipo le estaba pidiendo favores sexuales a una mujer. Denunciamos públicamente que este tipo estaba haciendo eso, pero a nosotras nos censuraron porque quienes estábamos ofreciendo eso éramos nosotras, dentro de su lógica era que nosotras éramos las que estamos ahí haciendo una acción incorrecta, que nosotras estábamos pidiendo de favor sexual a la mujer cuando había sido un vato (Entrevista Anónima, Artemisas Veterinarias).

Por otro lado, es común el insulto, las amenazas y la necesidad de mantener baja visibilidad dado que pueden volverse muy visibles por la misma lógica de la velocidad de Internet con el que se transmite la información, lo cual las hace vulnerables a ser identificables dentro del movimiento y a ser criminalizadas por los medios de comunicación y el Estado. Esto exige la necesidad de tener que estar alertas a falsas solicitudes de ayuda, "pero también nos llegan muchas, de bots, muchas cuentas falsas y, de repente, tenemos que estar haciendo esta depuración" (Patricia Rodríguez, Red de movimientos feministas de Guanajuato). Otro de los riesgos del activismo feminista en los espacios digitales es referente a la información que se comparte:

No sabes nunca quién está detrás de esa colectiva; por ejemplo, nosotros después del \#9N del 2020, hubo convocatorias de colectivas que se acaban de formar para hacer marchas que no estaban llamando a lo pacífico, ni siquiera teniendo los mínimos protocolos de seguridad; nosotros cuando convocamos a marchas tenemos un protocolo de seguridad, tanto interno, como externo para que cualquiera que vaya nuestra marcha tenga garantía de su seguridad y pedimos permiso (Tania Ramírez, Siempre Unidas).

Otro de los riesgos más comunes en las prácticas comunicativas en las plataformas digitales de las colectivas feministas se refiere al 'agotamiento emocional' al que las activistas están expuestas, que en el contexto del confinamiento se ha incrementado considerablemente (Portillo y Beltrán, 2021; Mendes et al., 2019) ante lo cual, en el caso de la colectiva Abogadas con Glitter, han tenido que emplear estrategias de autocuidado para preservar su integridad emocional y física en el uso de las plataformas digitales, la colectiva manifiesta: 
A nosotras nos afecta el entorno a pesar de estar atrás de la página de abogadas, somos mujeres y nos afecta innegablemente la situación que está ocurriendo con muchas compañeras, nos afecta el día a día porque puedes salir al metro, de pronto te acosan [...] es una situación alarmante, en alguna ocasión sumado como a este contexto produce un cansancio emocional de la situación de violencia y por otro lado como un hartazgo de que también en nuestra página de pronto nos llegan muchas agresiones y somos humanas y las sentimos, [...] decidimos sacar un pequeño comunicado [...] que se compartió mucho, la comentaron también, con mensajes de no están solas, las entendemos, las queremos, les agradecemos el apoyo que nos dan y donde también se invitaban esos comentarios a otras compañeras a que nos dieran un trato digno eso es lo que pedíamos dignificar nuestra labor y fue algo también muy significativo (Abogadas con Glitter).

Toda esta serie de agresiones y violencia en línea durante la pandemia se ha incrementado lo que ha conducido a las colectivas a desarrollar habilidades y estrategias de autocuidado digital para preservar su integridad física y emocional en el espacio digital.

\section{Conclusiones}

A partir del presente estudio podemos dar cuenta que las prácticas comunicativas en las redes sociodigitales de las colectivas feministas en México se insertan dentro del feminismo de la cuarta ola (Varela, 2020; Zimmerman, 2017), caracterizado por las transformaciones tecnológicas, la interseccionalidad y las posibilidades que ello permite para combinar diferentes prácticas comunicativas feministas que han generado una apertura para la creación de redes horizontales y democratización del feminismo (Laudano, 2019) a partir de su diversidad y de las tácticas que han empleado para hacerlo más asequible para las mujeres en un contexto de incremento de la violencia contra las mujeres en México en el contexto de la pandemia de la Covid-19, permitiendo una democratización del movimiento feminista, algunas colectivas de la Ciudad de México, ubican una última ola de movilizaciones feministas a partir del 24 de abril de 2016, con la llamada Primavera Violeta; sin embargo, persiste una tensión entre esta visión optimista sobre el movimiento feminista y la exclusión de miles de mujeres por la llamada brecha digital en el acceso a las diversas herramientas tecnológicas (Mazón, 2021), donde uno de los retos hacia el interior de las colectivas feministas ha sido la necesidad de profesionalización para la apropiación de plataformas digitales, como la alfabetización digital (Álvarez-Enríquez, 2020) y al mismo tiempo que estas herramientas son indispensables para la realización de los acompañamientos y asesorías para mujeres, teniendo en cuenta que la falta de acceso de todas las mujeres a estas plataformas digitales, es una preocupación de las colectivas entrevistadas.

Acerca de los hallazgos principales a partir de los datos empíricos de la presente investigación, sobre la realización de productos comunicativos como pueden 
ser infografías, comunicados, transmisiones en vivo y podcasts, por mencionar los más comunes. En su mayoría, las colectivas feministas no cuentan con recursos económicos, ya que son autogestivas y aprenden de forma autodidacta sobre el manejo de diversas plataformas o herramientas para realizar sus propios contenidos y diseños; o bien, piden ayuda de alguna conocida o conocido que puedan apoyarles de manera pro-bono. Esta situación, sí representa una desventaja frente a colectivas feministas que cuentan con una integrante profesional en la gestión de la comunicación digital, pues el impacto que pueda tener un mensaje, campaña o estrategia comunicativa es mucho mayor al hecho de no contar con los conocimientos para realizarlo.

Asimismo, los datos empíricos dan cuenta de la importancia del anonimato por los riesgos que implica estar bajo vigilancia por la criminalización de los medios tradicionales de comunicación que han expuesto sus perfiles y la necesidad del autocuidado hacia el interior de la colectiva por los constantes ataques, particularmente, en México existe un vacío de literatura que dé cuenta a profundidad sobre los riesgos que enfrentan las colectivas feministas en un contexto particular de inseguridad extendida por todo el territorio mexicano. Finalmente, las colectivas feministas resisten desde sus prácticas comunicativas digitales frente a la censura que las plataformas digitales imponen y la intensificación de la misoginia, trolls y ataques en sus redes sociodigitales bajo el contexto de la pandemia Covid-19, así como el incremento en la solicitud de ayuda en los espacios digitales por el aumento de violencia contra las mujeres.

Uno de los retos a futuro sobre la investigación de las prácticas comunicativas de las colectivas feministas en México gira con relación a profundizar sobre las estructuras y formas de actuar de los algoritmos en las plataformas digitales y las limitaciones que impactan en la organización, visibilización, socialización e interacciones comunicativas dentro de las colectivas feministas. Asimismo, queda pendiente para la investigación indagar sobre el aspecto estructural de las plataformas y de qué manera esto ha generado consecuencias para el activismo digital feminista en este entorno del capitalismo de vigilancia y fenómenos como el filtro burbuja o cámaras de eco, que pueden considerablemente repercutir en las prácticas comunicativas digitales de las colectivas feministas en México.

\section{Agradecimientos:}

Agradezco al Consejo Nacional de Ciencia y Tecnología (CONACyT) por su apoyo para la Estancia Posdoctoral por México 2021 y al Sistema Nacional de Investigadores (SNI), que se realiza en la Universidad de Guanajuato, bajo la asesoría de la Dra. Ericka López Sánchez en la Maestría de Análisis Político del Departamento de Estudios Políticos y Gobierno.

\section{Referencias}


Acosta, M., y Lassi, A. (2020). \#8M 2019. La conversación digital durante la Huelga Internacional de Mujeres. Revista Arbitrada Interdisciplinaria Koinonía, 5(9), 86-109.

Alvarado, M., Cruz Hernández, D. T., y Coba Mejía, L. (2020). Feminismos en movimientos en América Latina y el Caribe. Intersecciones entre pensamiento y acción política. Millcayac - Revista Digital De Ciencias Sociales, 7(12), 1124. https://revistas.uncu.edu.ar/ojs/index.php/millca-digital/article/view/3016

Álvarez-Enríquez, L. (2020). El movimiento feminista en México en el siglo XXI: juventud, radicalidad y violencia. Revista Mexicana de Ciencias Políticas y Sociales Universidad Nacional Autónoma de México, Nueva Época, Ixv, (240), 147-175.

Arteta, I. (2021, 26 de enero). En 2020, cada hora hubo 25 denuncias por violencia familiar. Animal Político. https://www.animalpolitico.com/2021/01/2020-cadahora-hubo-25-denuncias-violencia-familiar/.

Ayala, G. (2021, 10 de marzo). El paro nacional del 9 M tuvo un impacto de 37,000 millones de pesos. Milenio.

https://www.milenio.com/especiales/9-impacto-37-000-millones-pesos.

Banet-Weiser, S. y Miltner, K. (2016). \#Masculin-itySoFragile: Culture, structure, and networked misogyny. Feminist Media Studies, 16(1), 171-174. DOI: 10.1080/14680777.2016.1120490.

Becerril Martínez, W. (2019). Mujeres que colectivizan. Análisis de las apropiaciones tecnológicas de Internet que realizan las colectivas feministas. De Este Lado. Revista feminista de divulgación científica, año 2, núm. 4, enero-junio p. 1326 UNAM

Binder, I. (2019). Identidad y agencia colectiva del movimiento ciberfeminista en América Latina. El caso de ciberfeministaslatam. Dígitos: Revista de Comunicación Digital, (5), 210-233.

Castellanos, L. (2020, 6 de marzo). La rabia de las jóvenes feministas cimbra al gobierno de AMLO. Washington Post. https://www.washingtonpost.com/es/post-opinion/2020/03/05/la-rabia-de-lasjovenes-feministas-cimbra-al-gobierno-de-amlo.

Cerva-Cerna, D. (2021). Criminalización de la protesta feminista: el caso de las colectivas de jóvenes estudiantes en México, Revista de Investigaciones Feministas, 12(1), 115-125.

Cerva-Cerna, D. (2020). La protesta feminista en México. La misoginia en el discurso institucional y en las redes sociodigitales. Revista Mexicana de Ciencias Políticas $\quad y \quad$ Sociales, 65(240), 177-205. https://dx.doi.org/10.22201/fcpys.2448492xe.2020.240.76434.

Di Napoli, P. N. (2021). Jóvenes, activismos feministas y violencia de género en la UNAM: genealogía de un conflicto. Revista Latinoamericana de Ciencias $\begin{array}{lllll}\text { Sociales, } \quad \text { Niñez } & y & \text { Juventud, } & \text { 19(2), }\end{array}$ https://dx.doi.org/10.11600/rlcsnj.19.2.4567.

Equipo Editorial. (2020, 13 de julio). "El Gobierno de México está desarmando los mecanismos de protección a la mujer". Mi punto de vista. 
http://www.mipuntodevista.com.mx/el-gobierno-de-mexico-esta-desarmandolos-mecanismos-de-proteccion-a-la-mujer/.

Fotopoulou, A. (2016). Digital and networked by default? Women's organisations and the social imaginary of networked feminism. New Media y Society, 18(6), 9891005. https://doi.org/10.1177/1461444814552264.

Instituto Nacional de Geografía y Estadística (INEGI). (2021, 26 de enero). Datos preliminares revelan que de enero a junio de 2020 se registraron 17123 homicidios.

https://www.inegi.org.mx/contenidos/saladeprensa/boletines/2021/EstSociode mo/Defcioneshomicidio_En-Jun2020.pdf.

James, N., y Busher, H. (2012). Internet Interviewing" En J. Gubrium, J. Holstein, A. Marvasti, M. K. McKinney (eds.), Handbook of Interview Research, 2nd ed. SAGE publications.

Klandermans, B., y Staggenborg, S. (2002). Methods of social movement research. University of Minnesota Press.

Larrondo M., y Ponce C. (2019). Activismos Feministas Jóvenes en América Latina. Dimensiones y Perspectivas Conceptuales. En M. Larrondo y C. Ponce (eds.), Activismos feministas jóvenes. Emergencias, actrices y luchas en América Latina. CLACSO. (pp. 21-38). https://www.jstor.org/stable/j.ctvt6rkfs.

Laudano, C. (2019). Ciberfeminismo. En S. Gamba (coord.), Se va a caer. Conceptos básicos de los feminismos (pp. 65-70). Pixel.

Mazón, A. (2021, mayo-agosto). Mujeres en redes de lucha: ciberfeminismo como movimiento social contemporáneo, Revista Estudios Políticos, (53), 31-43. http://dx.doi.org/10.22201/fcpys.24484903e.2021.53.79429.

Mendes, K., Ringrose, J., y Keller, J. (2019). Digital feminist activism: Girls and women fight back against rape culture. Oxford University Press.

Núñez, S., Vázquez, S., y Fernández, D. (2016). Ciberfeminismo contra la violencia de género: análisis del activismo online-offline y de la representación discursiva de la víctima, Estudios sobre el Mensaje Periodístico, 22(2), 861877.

Pedraza, C., Rodríguez Cano, C. A. (2019). Resistencias sumergidas. Cartografía de la tecnopolítica feminista en México. Teknokultura. Revista de Cultura Digital y Movimientos Sociales, 16(2), 197-212.

Pfleger, S. (2021, marz- agosto). Fuertes, libres, rebeldes. Hacia una identidad más agentiva del movimiento feminista en México. Millcayac - Revista Digital de Ciencias Sociales, 8(14), 325-348. https://revistas.uncu.edu.ar/ojs3/index.php/millca-digital/article/view/4225.

Piñeiro-Otero, T. (2021). 'Escúchanos, hermana'. Los podcast como prácticas y canales del activismo feminista. Revista Inclusiones, 8(num. esp.), 231-254.

Piñeiro-Otero, T., y Martínez-Rolán, X. (2016). Los memes en el activismo feminista en la Red. \#ViajoSola como ejemplo de movilización transnacional. Cuadernos.info, (39), 17-37. DOI: 10.7764/cdi.39.1040.

Portillo Sánchez, M., y Beltrán Fuentes, D. (2020). Efectos de la pandemia por la Covid-19 en las movilizaciones feministas de la Ciudad de México. Revista 
Mexicana de Estudios de los Movimientos Sociales, 5(1), 6-36. http://www.revistamovimientos.mx/ojs/index.php/movimientos/article/view/250.

Ragin, C., y Amoroso, L. (1994). Constructing social research: The Unity and Diversity of Method. London: SAGE publications.

Registro Nacional de Personas Desaparecidas y No Localizadas en México (RNPDNO).

https://versionpublicarnpdno.segob.gob.mx/Dashboard/ContextoGeneral.

Reis, J., y Natansohn, G. (2019). Del ciberfeminismo al hackfeminismo: Notas para pensar Internet en tiempos de la algoritmia. En A. Rivoir y M. Morales (eds.), Tecnologías digitales: Miradas críticas de la apropiación en América Latina (pp. 391-406). CLACSO. DOI:10.2307/j.ctvt6rmh6.26.

Reverter, S., y Medina Vicent, M. (2020). El feminismo en 35 hashtags. Los Libros de La Catarata.

Revilla Blanco, M. (2019). "Del ¡Ni una más! al \#NiUnaMenos: movimientos de mujeres y feminismos en América Latina", Política y Sociedad, 56(1), pp. 4767.

Rovira-Sancho, G. (2018). El devenir feminista de la acción colectiva: las redes digitales y la política de prefiguración de las multitudes conectadas. Teknokultura: Revista de Cultura Digital y Movimientos Sociales, 15(2), 223-240.

Secretariado Ejetcutivo del Sistema Nacional de Seguridad Pública, Reportes de incidencia delictiva al mes de diciembre 2020 (Nueva metodología). Cifras de Víctimas del Fuero Común, 2015 - diciembre 2020. https://drive.google.com/file/d/1IAsT34UrJ85b4z8RkQGYlt8yU5c0rMPS/view.

Secretariado Ejecutivo del Sistema Nacional de Seguridad Pública (30 de abril de 2021) Información sobre violencia contra las mujeres. Incidencia delictiva y llamadas de emergencia 9-1-1, Centro Nacional de Información. https://drive.google.com/file/d/16iSlf8WBmnlrUEsaKWyey7_mJt2AMFSE/view

Sundén, J., y Paasonen, S. (2019). Inappropriate laughter: Affective homophily and the unlikely comedy of \#MeToo. Social Media + Society, 5(4). DOI: $10.1177 / 2056305119883425$.

Varela, N. (2020). El tsunami feminista. Nueva sociedad, (286), 93-106.

Observatorio Ciudadano Nacional de Feminicidio. (2021, 23 de febrero). Violencia contra las Mujeres en México. Informe del OCNF, CDD y REDTDT al Comité CEDAW. https://www.observatoriofeminicidiomexico.org/post/violencia-contralas-mujeres-en-m\%C3\%A9xico-informe-del-ocnf-cdd-y-redtdt-alcomit\%C3\%A9-cedaw.

Villar-Aguilés, A., y Pecourt Gracia, J. (2021). Antifeminismo y troleo de género en Twitter. Estudio de la subcultura trol a través de \#STOPfeminazis. Teknokultura. Revista de Cultura Digital y Movimientos Sociales, 18(1), 33-44. Zimmerman, T. (2017). \#Intersectionality: The fourth wave feminist Twitter community. Atlantis: Critical Studies in Gender, Culture y Social Justice (38), 1. https://journals.msvu.ca/index.php/atlantis/article/view/4304/54-70\%20PDF. 